\title{
Science of Synthesis: Applications of Domino Transformations in Organic Synthesis, Workbench Edition, 2 Vol.
}

\author{
Edited by Scott A. Snyder, 1st ed., Thieme: Stuttgart, 2016, 1284 pp, € 449, ISBN: 9783132401631
}

Applications of Domino Transformations in Organic Synthesis is a two-volume series in the Thieme Science of Synthesis (SOS) series, edited by Scott A. Snyder and published in May 2016. This latest workbench addition to the SOS library seeks to organise the vast literature on domino reactions into logical and manageable categories, summarise the major contributions within each defined category, provide experimental procedures for the most widely applicable reactions, and inspire readers to recognise opportunities for further investigations. It is a significant achievement of the authors and editor to have published a reference that succeeds admirably on all these fronts.

This reference work is divided into twenty-five detailed chapters written by contemporary experts in the field. Nominally, classification between sections is based on the process that initiates the domino sequence, but this requirement is often (and reasonably) relaxed to the presence of a process as a key step, to allow the emergence of themes within chapters. This effective approach to navigating the challenging and diverse area of domino reactions has been successfully employed in other reviews, and is accessible to both a general reader and one wishing to locate particular examples. Chapters are mostly focussed on reactions, including cationic polyene cyclisations, Diels-Alder reactions, and organocatalysed additions to $\mathrm{C}=\mathrm{C}$ bonds; whilst additional segments focus on processes promoted by general reagent classes like Brønsted and Lewis acids/bases, or specifics such as palladium- and dirhodium-containing reagents. Repetition between chapters with overlapping themes is minimized and where it does occur still serves to present topics in different contexts. Readers can promptly hone in on examples or sections for browsing by scanning the abstracts, detailed contents pages, or keyword and author indexes. The abstracts are of particular note, featuring edifying general schemes that allow the reader to quickly grasp the diversity of strategies and reactions that have been deployed.
Most chapters place an emphasis on, or reserve a section devoted to, examples that have found application in total synthesis. Work of the highest quality and most lasting impact is presented; readers interested only in the most recent trends in the field should look elsewhere. Mechanisms are generally not included in the schemes but key intermediates are drawn and the discussion is sometimes informed by mechanistic and physical chemistry considerations. Some chapters contain additional useful information such as detailed analysis of selectivity and perspectives on industrial applications. The very best chapters convey not only information but also a sense of appreciation for the often astonishing work featured, and an excitement at the significant and as yet unrealised potential in this area. This is a clearly defined ambition of the work and Snyder should be pleased that many authors have taken on this task and clearly hinted at the limitations of even the best examples.

As an illuminative discourse on domino reactions, the volumes will serve to impress and intrigue readers new to the field. Advanced researchers will find the breadth and complexity of reactions presented highly engaging and informative, and should be stimulated with new ideas and to delve further into the existing literature. The enthusiasm of the editor and authors for the subject is apparent and enhances the appeal of the text whilst in no way clouding methodical and critical analysis. Those working on a featured reaction type will benefit from seeing similar work put in context, perhaps realising new conceptual links. As with all the volumes in the SOS series, the books are particularly informative to laboratory chemists, or supervisors, since included experimental protocols quickly demonstrate the practicality of highlighted methods. This educational and stimulating work will make an invaluable contribution to both the reference library of a synthetic laboratory, and the personal collections of those with an appreciation for some of the finest examples of organic synthesis.

Nicholas J. Green and Andrew L. Lawrence, University of Edinburgh, UK 